\title{
Comment
}

\section{OR and the Social Sciences}

In the autumn of 1964 the Operational Research Society (UK) ran an international conference on "Operational Research and the Social Sciences". Proceedings are available (J.R. Lawrence, Ed.: Operational Research and the Social Sciences, Tavistock, London 1966). 25 years later, a second conference on this topic will be held in Cambridge, England, 10th-13th April 1989.

Is this a proper thing to do, to bring such separate fields of expertise together? - as separate as OR on the one hand and the social sciences on the other? What do they have in common?

To begin with quite a suitable definition of the field: "operations research is concerned with scientifically deciding how to best design and operate man-machine systems, usually under conditions requiring the allocation of scarce resources" (ORSA, Careers in OR, circa 1976). "Man-machine systems", "to best design and operate", "deciding", "allocation of scarce resources" do these terms not belong to the domain of the social sciences? Where else! Indeed, many branches of the social sciences are required for the interdisciplinary characteristics of an OR approach. "Deciding how to best design and operate man-machine systems..."

- without psychology would mean to do without a thorough understanding of the individual, his needs, his motives, his desires, his abilities, his cognitive culture;

- without group psychology and sociology would mean to do without a thorough understanding of group dynamics, group behaviour, group pressures, group structures, group leadership;
- without organizational sociology and organizational theory would mean to do without a thorough understanding of formal and informal structures of social systems; and

- without leadership theory would mean to do without a thorough understanding of how to guide and move social systems through personality, be it charisma, example, idol, power or brute force.

As Boothroyd (in his book, Articulate Intervention, London 1978) and others put it: OR/MS is intervention into management processes, participation in problem-solving and decision-making, as well as interaction with leaders. Is there a chance to fulful this role properly without the expertise of the social sciences?

Certainly, the image of the social sciences is colourful; certain branches of the social sciences have turned into shallow ideologies and other branches maintain their monodisciplinary virginity and keep away from any attempt at interdisciplinary co-operation, the historic virtue of $O R$. Let them stay away. We cannot and will not attempt to force any branches of the social sciences into a co-operation with $O R$ - just as we should not attempt to force branches of our own community into any kind of co-operation with the social sciences. Nonetheless, it remains a fascinating challenge to stimulate a new round of cross-fertilization between OR and social sciences, not only for the sake of OR or the social sciences, but mainly for the sake of advanced and succesful "deciding how to best design and operate man-machine systems".

HEINER MÜLLER-MERBACH 